\title{
SealWasteSafe: materials technology, monitoring techniques, and quality assurance for safe sealing structures in underground repositories
}

\author{
Vera Lay, Franziska Baensch, Sergej Johann, Patrick Sturm, Frank Mielentz, Prathik Prabhakara, \\ Detlef Hofmann, Ernst Niederleithinger, and Hans-Carsten Kühne
}

Bundesanstalt für Materialforschung und -prüfung (BAM), 12205 Berlin, Germany

Correspondence: Vera Lay (vera.lay@bam.de)

Published: 10 November 2021

\begin{abstract}
Within the project SealWasteSafe, we advance construction materials and monitoring concepts of sealing structures applied for underground disposal of nuclear or toxic waste. As these engineered barriers have high demands concerning integrity, an innovative alkali-activated material (AAM) is improved and tested on various laboratory scales. This AAM has low reaction kinetics related to a preferential slow release of the heat of reaction in comparison to alternative salt concretes based on Portland cement or magnesium oxychloride cements. Hence, crack formation due to thermally induced strain is reduced. After successful laboratory scale analysis (Sturm et al., 2021), the AAM is characterised on a larger scale by manufacturing test specimens (100$300 \mathrm{~L})$.

Conventional salt concrete (DBE, 2004) and the newly developed AAM are compared using two specimen geometries, i.e. cylindrical and cuboid. A comprehensive multisensor monitoring scheme is developed to compare the setting process of AAM and salt concrete for these manufactured specimens. The analysed parameters include temperature and humidity of the material, acoustic emissions, and strain variations. Passive sensor systems based on radiofrequency identification technology (RFID) embedded in the concrete, enable wireless access to temperature and humidity measurements and are compared to conventional cabled systems. Additionally, fibreoptic sensors (FOS) are embedded to record strain, but also have potential to record temperature and moisture conditions. Part of this project aims at demonstrating the high reliability of sensors and also their resistance to highly alkaline environments and to water intrusion along cables or at sensor locations. Further technical improvements were implemented so that first results clearly show the scalability of the setting process from previous small-scale AAM experiments and particularly the high potential of the newly developed approaches.

Furthermore, ultrasonic methods are used for quality assurance to detect obstacles, potential cracks and delamination. On the one hand, both active and passive ultrasonic measurements complement the results obtained from the multisensor monitoring scheme for the produced specimens. On the other hand, the unique large aperture ultrasonic system (LAUS) provides great depth penetration (up to nearly $10 \mathrm{~m}$ ) and can thus be applied at in situ sealing structures built as a test site in Morsleben by the Federal Company for Radioactive Waste Disposal (Bundesgesellschaft für Endlagerung, BGE) as shown by Effner et al. (2021). An optimised field layout identified from forward modelling studies and advanced imaging techniques applied to the measured data will further improve the obtained results. To characterise the inside of the test engineered barrier and achieve a proof-of-concept, an ultrasonic borehole probe is developed to enable phased arrays that can further improve the detection of potential cracks. Modelling results and first analysis of semispherical specimens confirmed the reliability of the directional response caused by the phased arrays of the newly constructed ultrasonic borehole probe.

Overall, the project SealWasteSafe improves the construction material, multisensor monitoring concepts and ultrasonics for quality assurance. This will help to develop safe sealing structures for nuclear waste disposal. The outcomes are particularly valuable for salt as a host rock but partly also transferrable to alternative conditions.
\end{abstract}


Kurzfassung. Innerhalb des Projekts SealWasteSafe wird die Entwicklung von Baustoffen und Überwachungskonzepten für Verschlussbauwerke mit Einsatz in der unterirdischen Lagerung von nuklearen oder toxischen Abfällen vorangetrieben. Da für solche technischen Barrieren hohe Anforderungen an die Integrität bestehen, wird ein innovatives alkaliaktiviertes Material (AAM) verbessert und in verschiedenen Labormaßstäben getestet. Dieses AAM weist eine niedrige Reaktionskinetik in Zusammenhang mit der präferierten langsamen Freisetzung von Reaktionswärme im Vergleich zu alternativen Salzbetonen auf der Grundlage von Portland-Zement oder Magnesium-Oxychlorid-Zementen auf. Infolgedessen ist die Entstehung von Rissen aufgrund von thermisch induzierter Verformung vermindert. Nach erfolgreicher Untersuchung im Labormaßstab (Sturm et al., 2021) wird das AAM nun in einem größeren Maßstab durch Herstellung von Prüfkörpern charakterisiert (100-300 L). Herkömmlicher Salzbeton (DBE, 2004) und das neu entwickelte AAM werden anhand von 2 Probegeometrien verglichen, d.h. zylindrisch und würfelförmig. Ein umfassendes multisensorisches Überwachungssystem befindet sich in Entwicklung, um damit den Abbindevorgang von AAM und Salzbeton für die hergestellten Prüfkörper zu vergleichen. $\mathrm{Zu}$ den ausgewerteten Parametern gehören Temperatur und Feuchtigkeit des Materials, akustische Emissionen sowie Dehnungsschwankungen. Passive Sensorsysteme auf der Grundlage der Radiofrequenzidentifizierungstechnologie (RFID), die in den Beton eingebettet sind, ermöglichen einen drahtlosen Zugang für Temperatur- und Feuchtigkeitsmessungen und werden mit herkömmlichen kabelgebundenen Systemen verglichen. Darüber hinaus sind faseroptische Sensoren (FOS) eingebettet, um Verformungen zu erfassen, sie verfügen aber auch über die Möglichkeit, Temperatur- und Feuchtigkeitsbedingungen aufzuzeichnen. Ein Teil dieses Projekts dient dem Ziel, die hohe Zuverlässigkeit der Sensoren und auch ihre Widerstandsfähigkeit gegenüber hochalkalischen Umgebungen und dem Eindringen von Wasser entlang von Kabeln oder an Sensorstandorten nachzuweisen. Weitere technische Verbesserungen wurden umgesetzt, sodass die ersten Ergebnisse deutlich die Skalierbarkeit des Abbindevorgangs aus den vorangegangenen AAM-Versuchen kleineren Maßstabs und insbesondere das hohe Potenzial der neu entwickelten Ansätze zeigen.

Außerdem werden Ultraschallverfahren für die Qualitätssicherung zum Erkennung von Störstellen, möglichen Rissen und der Ablösung von Schichten eingesetzt. Einerseits werden die Ergebnisse aus dem multisensorischen Überwachungssystem für die hergestellten Prüfkörper sowohl durch aktive als auch durch passive Ultraschallmessungen ergänzt. Andererseits ermöglicht das einzigartige Ultraschallsystem mit großer Apertur (LAUS) die Durchdringung großer Tiefen (bis zu beinahe $10 \mathrm{~m}$ ) und kann daher bei an Ort und Stelle befindlichen Verschlussbauwerke angewendet werden, die von der Bundesgesellschaft für Endlagerung (BGE) als Versuchsanlage in Morsleben errichtet wurden, wie von Effner et al. (2021) beschrieben. Eine optimierte Feldaufteilung, die anhand von Studien zur Vorwärtsmodellierung ermittelt wurde, und fortgeschrittene Bildgebungsverfahren, die auf die Messdaten angewendet werden, können darüber hinaus zur Verbesserung der bisher erhaltenen Ergebnisse beitragen. Zur Charakterisierung des Inneren der zu prüfenden technischen Barriere und zur Erlangung eines Proof-of-Concept wird eine Ultraschall-Bohrloch-Sonde entwickelt, mit der eine PhasedArray-Untersuchung möglich ist, die zur weiteren Verbesserung der Erkennung möglicher Risse führen kann. Die Ergebnisse der Modellierung und die erste Auswertung halbkugelförmiger Prüfkörper bestätigten die Verlässlichkeit der gerichteten Antwort, die durch die Phased-Array-Technik der neu konstruierten UltraschallBohrloch-Sonde ausgelöst wurde.

Insgesamt werden durch das Projekt SealWasteSafe das Baumaterial, multisensorische Überwachungskonzepte und die Ultraschalltechnik für die Qualitätssicherung verbessert. Das wird dazu beitragen, sichere Verschlussbauwerke für die Entsorgung nuklearer Abfälle zu entwickeln. Die Ergebnisse sind insbesondere für Salz als Wirtsgestein wertvoll, aber zum Teil auch auf alternative Bedingungen übertragbar.

Financial support. This research has been supported by the Bundesanstalt für Materialforschung und -prüfung (TFPSealWasteSafe).

\section{References}

Deutsche Gesellschaft zum Bau und Betrieb von Endlagern für Abfallstoffe $\mathrm{mbH}$ (DBE): Verfüllmaterial für Strecken mit hohen Anforderungen - Materialeigenschaften und Materialkennwerte Salzbeton M2, Bundesamt für Strahlenschutz, Salzgitter, 2004.
Effner, U., Mielentz, F., Niederleithinger, E., Friedrich, C., Mauke, R., and Mayer, K.: Testing repository engineered barrier systems for cracks - challenge, Materialwiss. Werkst., 52, 19-31, https://doi.org/10.1002/mawe.202000118, 2021.

Sturm, P., Moye, J., Gluth, G. J., Vogler, N., Taffe, A., and Kühne, H. C.: Properties of alkali-activated mortars with salt aggregate for sealing structures in evaporite rock, Open Ceramics, 5, 100041, https://doi.org/10.1016/j.oceram.2020.100041, 2021. 